\title{
Factors Associated With Nonalcoholic Fatty Liver Disease in Obese Adolescents
}

\author{
Moushira Erfan Zaki ${ }^{{ }^{*}}$, Wafaa Ezzat ${ }^{2}$, Yasser A Elhosary ${ }^{2}$, Omnia M Saleh $^{2}$ \\ ${ }^{1}$ Biological Anthropology Department, Medical Research Division, National Research Centre, Cairo, Egypt; ${ }^{2}$ Internal \\ Medicine Department, Medical Research Division, National Research Centre, Cairo, Egypt
}

\begin{abstract}
Citation: Zaki ME, Ezzat W, Elhosary YA, Sale OM. Factors Associated With Nonalcoholic Fatty Liver Disease in Obese Adolescents. Maced $J$ Med Sci. 2013 Sep 15: 6(3):273-277.

Key words: NAFL; ultrasonography; obesity; adolescents; abdominal fat.

"Correspondence: Prof. Moushira Erfan Zaki. National Research Centre, El-Behooth St,Dokki, Giza, Cairo 12311, Egypt. E-Mail: moushiraz@yahoo.com

Received: 22-Jul-2013; Revised: 03-Aug2013; Accepted: 09-Aug-2013; Online first: 10-Sep-2013

Copyright: ๑ 2013 Zaki ME. This is an openaccess article distributed under the terms of the Creative Commons Attribution License, which permits unrestricted use, distribution, and reproduction in any medium, provided the original author and source are credited.

Competing Interests: The authors have declared that no competing interests exist.
\end{abstract}

\begin{abstract}
Background and Objectives: Nonalcoholic fatty liver disease (NAFLD) is a common cause of liver disease in children and adolescents. The aim of this study is to investigate the prevalence of NAFLD in a sample of obese adolescents and to identify the clinical and biochemical characteristics in subjects with NAFLD.

Design and Settings: A cross-sectional study was conducted on 366 adolescents. The data were collected from June 2011 to July 2012.

Patients and Methods: The study included 202 females and 164 males. All were subjected to anthropometric, biochemical and ultrasongraphic assessment.

Results: The prevalence of NAFLD in general was $57.65 \%(62.19 \%$ for males and $53.96 \%$ for females). Results showed statistical gender difference. There were statistical differences in BMI, WC, TG, LDL-C, HDL-C, body fat percentage (BF \%), subcutaneous fat layers, visceral fat and ALT between NAFLD group and non- NAFLD group in both genders. The prevalence of obesity was significantly higher in the NAFLD group than non NAFLD group. The present study showed that $58.29 \%$ of NAFLD were obese in which $49 \%$ of males and $67 \%$ of females.
\end{abstract}

Conclusion: Abdominal obesity and dyslipidemia are risk factors for NAFLD. Prevalence of NAFLD among obese adolescents is high and prevention is extremely important.

\section{Introduction}

Nonalcoholic fatty liver disease (NAFLD) represents a spectrum of conditions ranging from simple hepatic steatosis to potentially fatal nonalcoholic steato-hepatitis and cirrhosis. NAFLD may be the most common cause of liver disease in both adults and children. Concurrent with the epidemic of childhood obesity, pediatric NAFLD is a growing global problem [1]. The most mild form is simple steatosis in which triglyceride accumulates within hepatocytes. A more advanced form of NAFLD, nonalcoholic steatohepatitis (NASH), includes inflammation and liver cell injury. Several investigators have reported that NASH may present with cirrhosis and end-stage liver disease in some children. Established risk factors for NAFLD in adolescents include obesity, insulin resistance, and type 2 diabetes [2-4].

Obesity is a pro-inflammatory state that leads to insulin resistance (IR), which is closely associated with NAFLD development and progression. As the number of obese children has increased worldwide, risks for obesity-related metabolic and endocrine derangements, including hyperinsulinemia, hypertriglyceridemia, and hypercholesterolemia, have led to early development and increased incidence of type 2 diabetes, cardiovascular disease, hypertension and NAFLD [5-7]. NAFLD has a multifactorial etiology and 
a combination of environmental, genetic and metabolic factors play a role in the development of advanced disease. Genetic predisposition, overabundance of calorie-rich food and lack of physical activity contribute to development of obesity. So the pathophysiology of NAFLD is not fully understood.

Therefore, study of the prevalence of NAFLD in a sample of Egyptian Young Adults and identifying its risk factors would be critically important. The study attempts to assess the relationship between NAFLD and gender, obesity and lipids disturbance.

\section{Subjects and Methods}

A descriptive cross-sectional study was conducted on 366 adolescents 202 females and 164 males. Their mean age was $17.45 \pm 2.54$ years. The data were collected from June 2011 to July 2012 and were extracted from a project entitled "Obesity among Youth: Lifestyle and Genetic Factors" funded by the Science and Technology Development Fund (STDF), Egypt. This study protocol was approved by the ethical committee board of the National Research Centre of Egypt (No.10/223). An informed written consent was obtained from all participants. All individuals were clinically evaluated. The diagnostic of NAFLD was established by tests of liver function and ultrasonography followed by the exclusion of the secondary causes of hepatic steatosis: (1) alcohol intake of $30 \mathrm{~g} /$ day or more for males and $20 \mathrm{~g} /$ day or more for females, (2) Wilson disease, intestinal bypass surgery, glutenic entheropathy, (3) ingestion of drugs known to produce hepatic steatosis including methotrexate, tamoxifen, amiodarone, nucleoside analogues, (4) a positive serology for hepatitis B or C virus, (5) a history of another known liver disease. The subjects were interviewed to obtain their history of hypertension, diabetes mellitus, dyslipidemia, myocardial infarction, stroke, as well as alcohol consumption and smoking.

\section{Anthropometric measures}

Standing height without shoes was measured twice with a standard scale to the nearest $0.1 \mathrm{~cm}$. Height was measured with the patients standing with their backs leaning against the stadiometer of the same scale. Body weight was measured with the lightest clothing to the nearest $0.1 \mathrm{~kg}$ and $\mathrm{BF} \%$ was measured by Tanita Body Composition Analyzer (SC-330). BMI was calculated as weight in kilograms divided by height in meters squared $(\mathrm{kg} / \mathrm{m} 2)$. WC and hip circumference $(\mathrm{HC})$ were measured in $\mathrm{cm}$ using a plastic, non-stretchable tailor's tape. WC was measured with light clothing at a level midway between the lower rib margin and the iliac crest standing and breathing normally. $\mathrm{HC}$ was measured at the level at the widest circumference over the buttocks (at the greater trochanter). The waist hip ratio (WHR) was calculated as WC divided by HC. Obese adolescents had BMI greater than $95^{\text {th }}$ percentile for age and gender, according to the National Egyptian Growth Curves of Children and Adolescents [10].

\section{Laboratory measurements}

Blood samples were collected from all the participants after an overnight fast. Serum glutamatepyruvate transaminase (ALT), aspartate aminotransferase (AST), triglycerides (TG), cholesterol (TC), high density lipoprotein cholesterol (HDL-C), lowdensity lipoprotein cholesterol (LDL-C) and fasting plasma glucose level were measured through standard laboratory methods.

\section{Abdominal ultrasonographic examination}

Ultrasonography was carried out by using GE logic a 200-ultrasound machine. The linear array probe $(7.5 \mathrm{MHz})$ was used to measure the subcutaneous (SC) and Visceral fat (VF) abdominal fat layers. The subcutaneous minimum (SC min) and Visceral fat maximum (VF max) measurements were taken from the region just below the xyphoid process, whereas the subcutaneous maximum (SC max) and Visceral fat minimum (VF min) fat layers were measured from the region just above the umbilicus [8]. The convex-array probe $(3.5 \mathrm{MHz})$ was used for measuring visceral abdominal fat and anterior wall of the aorta [9]. The patients were asked to suspend respiration during examination and special care was taken to keep the probe just touching the skin to prevent compression of fat layers. A convex-array probe $(3.5 \mathrm{MHz})$ was used to demonstrate liver fatty infiltration. This was defined as increased echogenicity of the liver parenchyma without obvious mass effect and slightly impaired or poor visualization of the intrahepatic vessels and diaphragm. The patients were evaluated by the same radiologist in four groups according to the presence and severity of liver steatosis determined by ultrasonography. The changes of the echogenicity due to the fatty infiltration of liver parenchyma were evaluated according to the adjacent renal parenchyma.

\section{Statistical analyses}

All statistical analyses were performed using SPSS 16.0. Data is expressed as the mean \pm standard deviation. Differences between groups were tested using an independent two-sample t-test and chi-square test was used to test for differences in the distribution of categorical variables. P-values $<0.05$ were considered statistically significant.

\section{Results}

Off 366 the participants, $153(41.81 \%)$ were obese. The prevalence of NAFLD in obese 
adolescents was $80.39 \%$ (123 out of 153). As shown in Tables 1 and 2 the prevalence of NAFLD in general was $57.65 \%$ ( 211 out of 366 ), $62.19 \%$ for males (102 out of 164 ) and $53.96 \%$ for females (109 out of 202), with no significant gander difference between NAFLD and non NAFLD group. In the NAFLD group, $58.29 \%$ were obese (123 out of 211 cases) in which $49 \%$ in males and $67 \%$ in females. There was a statistical significant gender difference $(P<0.02)$.

Table 1: Comparison between male subjects with and without NAFLD.

\begin{tabular}{lccc}
\hline Variables & $\begin{array}{c}\text { Non NAFLD } \\
(\mathrm{n}=62)\end{array}$ & $\begin{array}{c}\text { NAFLD } \\
(\mathrm{n}=102)\end{array}$ & P-value \\
\hline Age $($ years $)$ & $17.64 \pm 3.51$ & $17.24 \pm 4.30$ & $<0.715$ \\
BMI & $20.32 \pm 5.54$ & $31.31 \pm 7.53$ & $<0.001$ \\
WC $(\mathrm{cm})$ & $67.92 \pm 15.19$ & $93.47 \pm 17.82$ & $<0.001$ \\
WHR & $0.80 \pm 0.80$ & $0.82 \pm 0.13$ & $<0.349$ \\
SCF $\min (\mathrm{cm})$ & $1.07 \pm 0.55$ & $2.0 \pm 0.62$ & $<0.001$ \\
SCF $\max (\mathrm{cm})$ & $1.162 \pm 0.7515$ & $2.6 \pm 1.02$ & $<0.001$ \\
VF $\min (\mathrm{cm})$ & $3.075 \pm 1.11$ & $4.6 \pm 1.5$ & $<0.001$ \\
VF $\max (\mathrm{cm})$ & $3.55 \pm 1.360$ & $5.64 \pm 1.79$ & $<0.001$ \\
Liver size $(\mathrm{cm})$ & $11.58 \pm 1.63$ & $15.7 \pm 1.29$ & $<0.001$ \\
Spleen size $(\mathrm{cm})$ & $8.92 \pm 1.31$ & $8.86 \pm 0.79$ & $<0.808$ \\
BF $\%$ & $11.21 \pm 7.18$ & $27.80 \pm 13.06$ & $<0.001$ \\
Glucose $(\mathrm{mg} / \mathrm{dL})$ & $86.68 \pm 13.95$ & $96.73 \pm 38.69$ & $<0.246$ \\
Cholesterol $(\mathrm{mg} / \mathrm{dL})$ & $154.55 \pm 31.450$ & $175.00 \pm 46.15$ & $<0.080$ \\
Triglycerides $(\mathrm{mg} / \mathrm{dL})$ & $69.60 \pm 36.0$ & $113.85 \pm 76.67$ & $<0.018$ \\
HDL-C $(\mathrm{mg} / \mathrm{dL})$ & $62.11 \pm 15.36$ & $50.02 \pm 19.192$ & $<0.032$ \\
LDL-C $(\mathrm{mg} / \mathrm{dL})$ & $94.13 \pm 16.62$ & $105.03 \pm 27.22$ & $<0.051$ \\
AST $(\mathrm{U} / \mathrm{L})$ & $27.82 \pm 15.196$ & $31.05 \pm 18.219$ & $<0.482$ \\
ALT $(\mathrm{U} / \mathrm{L})$ & $17.95 \pm 19.08$ & $30.70 \pm 29.96$ & $<0.046$ \\
Obesity $(\%)$ & $6.5(4 / 62)$ & $49(50 / 102)$ & $<0.001$ \\
\hline BMl: Body mass index: WHR: waist to hip ratio; SCF min: subcutaneous fat minimum:
\end{tabular}

BMl: Body mass index; WHR: waist to hip ratio; SCF min: subcutaneous fat minimum SCF max: subcutaneous fat maximum ; VF min: Visceral fat minimum; VF max: Visceral fat maximum; HDL: high density lipoprotein; LDL: low density lipoprotein; AST :aspartate transaminase, ALT: alanine transaminase

In males, the average age of the subjects with NAFLD was $17.24 \pm 4.30$, and $17.64 \pm 3.51$ in those without NAFLD. In females, the average age in NAFLD and non NAFLD group was $17.48 \pm 3.79$ and $16.98 \pm 2.88$ respectively. There were statistical differences in BMI, WC, TG, LDL-C, HDL-C, BF\%, visceral fat, subcutaneous fat and ALT between NAFLD group and non NAFLD group in both genders. In addition, NAFLD group had higher liver size. The prevalence of obesity in the NAFLD group was significantly higher than in non NAFLD group in both genders. There were no differences in WHR, spleen size, AST, glucose and cholesterol levels between NAFLD group and non NAFLD group.

Table 2: Comparison between female subjects with and without NAFLD.

\begin{tabular}{lccc}
\hline Variables & $\begin{array}{c}\text { Non NAFLD } \\
(\mathrm{n}=93)\end{array}$ & $\begin{array}{c}\text { NAFLD } \\
(\mathrm{n}=109)\end{array}$ & P-value \\
\hline Age (years) & $16.98 \pm 2.88$ & $17.48 \pm 3.794$ & $<0.342$ \\
BMI $(\mathrm{kg} / \mathrm{m} 2)$ & $25.53 \pm 6.52$ & $34.004 \pm 6.857$ & $<0.001$ \\
WC $(\mathrm{cm})$ & $78.75 \pm 2.28$ & $93.98 \pm 15.88$ & $<0.001$ \\
WHR & $0.76 \pm 0.177$ & $0.80 \pm 0.070$ & $<0.079$ \\
SCF $\min (\mathrm{cm})$ & $1.51 \pm 0.47$ & $2.24 \pm 0.71$ & $<0.001$ \\
SCF $\max (\mathrm{cm})$ & $1.89 \pm 0.68$ & $2.74 \pm 0.80$ & $<0.001$ \\
VF $\min (\mathrm{cm})$ & $3.18 \pm 1.22$ & $4.33 \pm 1.44$ & $<0.001$ \\
VF max $(\mathrm{cm})$ & $4.13 \pm 1.292$ & $5.31 \pm 1.685$ & $<0.001$ \\
Liver size $(\mathrm{cm})$ & $11.50 \pm 1.81$ & $14.91 \pm 1.98$ & $<0.001$ \\
Spleen size $(\mathrm{cm})$ & $8.73 \pm 0.8754$ & $8.60 \pm 0.96$ & $<0.342$ \\
BF \% & $26.80 \pm 10.78$ & $40.58 \pm 7.146$ & $<0.001$ \\
Glucose $(\mathrm{mg} / \mathrm{dL})$ & $89.93 \pm 10.11$ & $92.50 \pm 13.97$ & $<0.213$ \\
Cholesterol $(\mathrm{mg} / \mathrm{dL})$ & $180.46 \pm 35.80$ & $189.61 \pm 47.26$ & $<0.195$ \\
Triglycerides $(\mathrm{mg} / \mathrm{d})$ & $77.40 \pm 32.173$ & $96.84 \pm 41.787$ & $<0.002$ \\
HDL-C $(\mathrm{mg} / \mathrm{dL})$ & $62.44 \pm 17.41$ & $55.02 \pm 17.57$ & $<0.011$ \\
LDL-C $(\mathrm{mg} / \mathrm{dL})$ & $105.49 \pm 29.92$ & $113.31 \pm 40.86$ & $<0.045$ \\
AST $(\mathrm{mg} / \mathrm{dL})$ & $24.10 \pm 11.82$ & $24.77 \pm 12.55$ & $<0.736$ \\
ALT $(\mathrm{mg} / \mathrm{dL})$ & $15.19 \pm 10.46$ & $20.71 \pm 16.26$ & $<0.019$ \\
Obesity $(\%)$ & $28(26 / 93)$ & $67(73 / 109)$ & $<0.001$ \\
\hline
\end{tabular}

BMI: Body mass index; WHR: waist to hip ratio; SCF min: subcutaneous fat minimum SCF max: subcutaneous fat maximum : VF min: Visceral fat minimum; VF max: Viscera fat maximum; high density lipoprotein; LDL: low density lipoprotein; AST: aspartate transaminase, ALT: alanine transaminase

\section{Discussion}

Although the prevalence of these diseases varies significantly among ethnic groups, NAFLD is increasingly recognized as an important and common factor affecting approximately $20 \%$ of the general population [11]. Nowadays NAFLD has become a major public health hazard worldwide. The prevalence rates range from $24 \%$ to $42 \%$ in Western countries and $5 \%$ to $40 \%$ in Asian countries [12-14]. Hispanics having the highest prevalence (45-58\%), followed by Caucasians (33-44\%) and African Americans (24$35 \%$ ) [15-16]. Obesity might be seen in approximately $71 \%$ of patients with NAFLD [17]. In terms of free fatty acid (FFA) discharge and steatotic liver formation, visceral and central adipose tissues have more effective role in comparison with peripheral adipose tissue [18]. Abdominal fat layers comprise subcutaneous, preperitoneal and visceral fat compartments. The distribution of abdominal fat may be more important than total body fat.

Ultrasonographic fatty liver is defined when the accumulation of fat, mainly triglycerides, in hepatocytes exceeds $5 \%$ of the liver weight, and NAFLD refers to ultrasonographic fatty liver accompanied with liver function disruption [19].

The incidence of NAFLD has increased since the first case of pediatric non - alcoholic steatohepatitis (NASH) was described in 1983[20]. However, the actual prevalence of NAFLD and NASH in children remains largely unknown because of the lack of population-based studies and reliable noninvasive screening tools. Several studies in different countries selectively examined the prevalence of NAFLD in groups of obese children and found that it might range from $23 \%$ to $77 \%$ [21-22]. The study of el-karksi et al 2011 [23] showed that the estimated prevalence of NAFLD was $38.5 \%$ in obese Egyptian children and adolescents. The results of the present study showed higher values, the prevalence of NAFLD in general was $57.65 \%(62.19 \%$ for males and $53.96 \%$ for females). Obesity and insulin resistance are crucial for the pathogenesis of NAFLD [17]. The present study showed that $58.29 \%$ of NAFLD were obese (123 out of 211 NAFLD cases) in which $49 \%$ in males and $67 \%$ in females, with statistical gender difference $(P<0.02)$. In the National Health and Nutrition Examination survey conducted in the United States from 1988 to 1994, more than 20\% of the children aged 12 to 17 years were overweight (85th percentile of BMI in $\mathrm{kg} / \mathrm{m} 2$ for age) and $8 \%$ to $17 \%$ were obese (95th percentile of BMI for age) in different ethnic groups [24].

Among adolescents, $60 \%$ with elevated ALT levels were either overweight or obese, who, though having the modest alcohol consumption, were at an increased risk of developing obesity related liver disease [4].

Apart from obesity, several risk factors were reported to be associated with NAFLD; such as 
diabetes, insulin resistance (IR), and hypertriglyceridemia.

The present study emphasized the relationships between biochemical, anthropometric measurements and abdominal fat accumulation and presence of NAFLD in Egyptian adolescents. BMI was recommended as an index of peripheral obesity and waist circumference as one of indices of abdominal obesity. Previous studies reported that waist circumference is a good predictor of the visceral obesity and also increased according to the severity of liver steatosis [25, 26]. The potential source of lipid accumulation in the livers of patients with NAFLD is the fat stored in adipose tissue. Approximately $80 \%$ of fatty acid content of plasma nonesterified fatty acid concentrations (NEFA) pool is contributed by adipose tissue and BMI was progressively increased with the degree of steatosis, total fat mass (\%) increased in severe steatosis [27].

The present study supports the belief that obese Hispanic adolescents are more likely than obese non-Hispanic adolescents to develop fatty liver. This is consistent with data from the Third National Health and Nutrition Examination Survey showing that an elevated ALT is more common in obese American adults [28].

Gender difference in prevalence of obesity in subjects with NAFLD was noted in this study. With the increasing prevalence of obesity and type 2 diabetes mellitus in the general population, NAFLD has become a common diagnosis in clinical practice. There is increasing evidence that obesity, hyperglycemia and insulin resistance are risk factors for NAFLD in children and adolescents. Thus, it is of great importance to investigate the obese children and adolescents and to seek the sensitive index predicting early hepatic lesion and related dyslipidemia. It was found that there was no significant differences between NAFLD group and non NAFLD group as regards spleen size while Mendes and his colleagues [29] found that one hundred patients of 354 had portal hypertension at the time of NAFLD diagnosis (28.2\%), they used esophageal varices, splenomegaly, portosystemic encephalopathy, and ascites as noninvasive indicators for portal hypertension. In the current study, the mean values of spleen size were within normal range even in subjects with NAFLD which could be referred to younger ages of the studied subjects in current study so that, process of steatohepatitis and liver fibrosis had not occurred yet.

It was noticed that percentage of fatty liver among children was more than that of obese children. This means that fatty liver not only associated with overt obesity but also just overweight children can have enlarged liver.

Abdominal fat is composed of several distinct anatomic depots: subcutaneous fat, which can be divided into anterior and posterior or superficial and deep layers, and intraabdominal fat, which can be divided into intraperitoneal and retroperitoneal sites. Intraperitoneal fat, also known as visceral fat, is composed of mesenteric and omental fat masses. Although the absolute amount of each of these depots is much larger in upper-body obese than in lean persons, the relative amount of abdominal fat with respect to total body fat mass is often similar in both groups. For example, visceral fat constitutes about $10 \%$ of total body fat mass in lean and obese men (30). Thus, we cannot depend on BMI only to define obese persons but truncal obesity must be assessed to avoid associated problems like NAFLD.

In conclusion, the study suggests that abdominal obesity and dyslipidemia can increase the risk of NAFLD among Egyptian adolescents in both genders. Thus, obesity is a major risk factor and is associated with high rates of NAFLD, and it is of great importance to investigate the obese adolescents and seek for sensitive index predicting early hepatic lesion and related dyslipidemia.

\section{Acknowledgment}

Authors are greatly thankful to the Science and Technology Development Fund (STDF) for funding the project entitled "Obesity among Youth: Lifestyle and Genetic Factors" (1225) that enabled us to use the data to establish this work.

\section{References}

1. Janssen I, Katzmarzyk PT, Boyce WF, et al. Comparison of overweight and obesity prevalence in school-aged youth from 34 countries and their relationships with physical activity and dietary patterns. Obes Rev. 2005; 6(2):123-132.

2. Schwimmer JB, Deutsch R, Rauch JB, Behling C, Newbury R, Lavine JE. Obesity, insulin resistance, and other clinicopathological correlates of pediatric nonalcoholic fatty liver disease. J Pediatr. 2003; 143:500-505.

3. Franzese A, Vajro $P$, Argenziano A, et al. Liver involvement in obese children. Ultrasonography and liver enzyme levels at diagnosis and during follow-up in an Italian population. Dig Dis Sci . 1997; 42: 1428-1432.

4. Strauss RS, Barlow SE, Dietz WH. Prevalence of abnormal serum aminotransferase values in overweight and obese adolescents. J Pediatr. 2000; 136:727-733.

5. Must A, Strauss RS. Risks and consequences of childhood and adolescent obesity. Int J Obes Relat Metab Disord. 1999; 23(2):S2-11.

6. Freedman DS. Clustering of coronary heart disease risk factors among obese children. J Pediatr Endocrinol Metab. 2002; 15:1099 - 108.

7. Csa'bi G,Torok K, Jeges S, et al. Presence of metabolic cardiovascular syndrome in obese children. Eur J Pediatr. 2000; 159: 91 - 43 - 8

8. Matteoni CA, Younossi ZM, Gramlich T, Boparai N, Liu YC, McCullough AJ. Non-alcoholic fatty liver disease: a spectrum of clinical and pathological severity. Gastroenterology. 1999; 116(6): 1413-9.

9. Lee RG. Nonalcoholic steatohepatitis: tightening the morphological screws on a hepatic rambler. Hepatology. 1995; 21(6): 1742-3. 
10. Standard Egyptian Growth. Diabetes Endocrine Metabolism Pediatric Unit Cairo University Children's Hospital. [Last accessed on 2009 Aug 13, Last revised 2008 Nov 29]. Available from: http://www.dempuegypt.blogspot.com.

11. Ballentani S, Saccoccio G, Masutti $F$, et al. Prevalence of andrisk factors for hepatic steatosis in northern Italy. Ann Intern Med. 2000; 132:112 - 7.

12. Amarapurkar DN, Hashimoto E, Lesmana LA, Sollano JD, Chen PJ, Goh KL: How common is non-alcoholic fatty liver disease in the Asia-Pacificregion and are there local differences? J Gastroenterol Hepatol. 2007; 22(6):788-793.

13. Fan JG, Saibara T, Chitturi S, Kim BI, Sung JJ, Chutaputti $A$ : What are the risk factors and settings for non-alcoholic fatty liver disease in Asia-Pacific? J Gastroenterol Hepatol. 2007; 22(6):794-800.

14. Caballeria L, Auladell MA, Toran P, Miranda D, Aznar J, Pera G, Gil D, MunozL, Planas J, Canut S, et al: Prevalence and factors associated with the presence of non alcoholic fatty liver disease in an apparently healthyadult population in primary care units. BMC Gastroenterol. 2007; 7:41.

15. Williams CD, Stengel J, Asike MI, Torres DM, Shaw J, Contreras $M$, Landt CL, Harrison SA: Prevalence of nonalcoholic fatty liver disease and nonalcoholic steatohepatitis among a largely middle-aged population utilizing ultrasound and liver biopsy: a prospective study. Gastroenterology. 2011; 140(1):124-131.

16. Browning JD, Szczepaniak LS, Dobbins $R$, Nuremberg $P$ Horton JD, Cohen JC, Grundy SM, Hobbs HH: Prevalence of hepatic steatosis in an urbanpopulation in the United States: impact of ethnicity. Hepatology. 2004; 40(6):1387-1395.

17. Wanless IR, Lentz J. Fatty liver hepatitis (steatohepatitis) and obesity: an autopsy study with analysis of risk factors. Hepatology. 1990; 12(5): 1106-10.

18. Medina J, Fernandez-Salazar LI, Garcia-Buey L, MorenoOtero R. Approach to the pathogenesis and treatment of nonalcoholic steatohepatitis. Diabetes Care. 2004; 27(8): 2057-66.

19. Schreuder TC, Verwer BJ, van Nieuwkerk CM, et al. Nonalcoholic fatty liver disease: an overview of current insights in pathogenesis, diagnosis and treatment. World $J$ Gastroenterol. 2008; 14, 2474 - 86.

20. Moran JR, Ghishan FK, Halter SA, et al. Steatohepatitis in obese children: a cause of chronic liver dysfunction. Am J Gastroenterol. 1983; 78, 374 - 7.

21. Schwimmer JB, McGreal N, Deutsch R, et al. Influence ofgender, race, and ethnicity on suspected fatty liver in obese adolescents. Pediatrics. 2005; 115, e561 - 5.

22. Franzese $A$, Vajro $P$, Argenziano $A$, et al. Liver involvement in obese children. Ultrasonography and liver enzyme levels at diagnosis and during follow - up in an Italian population. Dig Dis Sci. 1997; 42: 1428 - 32.

23. el-Karaksy HM, el-Koofy NM, Anwar GM, el-Mougy FM, elHennawy A, Fahmy ME. Predictors of non-alcoholic fatty liver disease in obese and overweight Egyptian children: single center study. Saudi J Gastroenterol. 2011; 17(1):40-6

24. Sokol RJ. The chronic disease of childhood obesity: the sleeping giant has awakened. J Pediatr. 2000; 136: 711 - 3.

25. Jia WP, Lu JX, Xiang KS, Bao YQ, Lu HJ, Chen L. Prediction of abdominal visceral obesity from body mass index, waist circumference and waist-hip ratio in Chinese adults: receiver operating characteristic curves analysis. Biomed Environ Sci. 2003; 16(3): 206-11.

26. Kral JG, Schaffner F, Pierson RN Jr, Wang J. Body fat topography as an independent predictor of fatty liver. Metabolism. 1993; 42(5): 548-51.
27. Donnelly KL, Smith Cl, Schwarzenberg SJ, Jessurun J, Boldt MD, Parks EJ. Sources of fatty acids stored in liver and secreted via lipoproteins in patients with nonalcoholic fatty liver disease. J Clin Invest. 2005; 115(5): 1343-51.

28. Clark JM, Brancati FL, Diehl AM. The prevalence and etiology of elevated aminotransferase levels in the United States. Am J Gastroenterol. 2003; 98:960-967.

29. Mendes FD, Suzuki A, Sanderson SO, Lindor KD, Angulo P.. Prevalence and indicators of portal hypertension in patients with nonalcoholic fatty liver disease. Clin Gastroenterol Hepatol. 2012 ;10(9):1028-33.

30. Abate N, Burns D, Peshock RM, Stray-Gundersen J, and Grundy SM. Relationship of generalized and regional adiposity to insulin sensitivity in men. J Clin Invest. 1995; 96:88-98. 